Journal of Fisheries International 5 (1): 8-13, 2010

ISSN: 1817-3381

(C) Medwell Journals, 2010

\title{
Modeling of Aquatic Infectious Disease in Bangladesh
}

\author{
${ }^{1}$ Nandita Roy and ${ }^{2}$ Nilimesh Halder \\ ${ }^{1}$ Discipline of Agrotechnology, University of Khulna, Khulna, Bangladesh \\ ${ }^{2}$ Discipline of Computer Science and Engineering, University of Khulna, Khulna, Bangladesh
}

\begin{abstract}
Fishery is considered as an emerging farming sector in Bangladesh and plays an important role in annual economic growth. This emerging industry is facing several hindrances and infectious disease of fish is one of them. In early days, fish disease research was on various respects of pathogens rather than investigating dynamics of disease spread in aquatic population. Vast amounts of data are available on the responses of these pathogens to environmental and host related factors and generally statistical modeling of those data could be used to understand the relative importance of the factors influencing the spread of disease. In particular, the data obtained from aquaculture systems could be robust in formulating mathematical model due to fewer assumptions involved. Thus, nonlinear relationships between various factors affecting disease outcome can be evaluated more efficiently and realistic predictions can be obtained using mathematical model. In this study, we formulate an SIR epidemic model for aquatic infectious disease to investigate the disease dynamics. The goal of this study is to assess the basic concept of disease modeling for aquatic infectious disease and provide insight into the relative importance of parameters playing a major role in disease outbreaks.
\end{abstract}

Key words: Aquatic infectious disease, mathematical modeling, SIR mode, fishery, pathogens, Bangladesh

\section{INTRODUCTION}

Fish play an important role among the population in Bangladesh. Situated in the delta of the Brahmaputra, Meghna and Ganges rivers, the climate, water and soil conditions of Bangladesh are favorable for inland fisheries and aquaculture. According to the Bangladesh Bureau of Statistics (BBS), the fisheries sector including aquaculture and capture fisheries has had an annual growth exceeding $7 \%$ since 1995 and contributed $6 \%$ to the country's GDP in 2000 (Statistical Year book of Bangladesh), (Household Expenditure Survey, BBS, $2000 \mathrm{a}, \mathrm{b})$. With annual fish consumption of about $14 \mathrm{~kg}$ person $^{-1}$ in 2000 , fish account for $60-80 \%$ of the animal protein consumed by the population and also provide essential vitamins, minerals and fatty acids (Thompson et al., 2002).

Inland fisheries and freshwater aquaculture are the main source of these nutrients for most of the Bangladeshi populations. Over the last 2 decades, there has been a dramatic increase in inland freshwater aquaculture production. Department of Fisheries' statistics indicate that fish pond production in Bangladesh increased sharply from 123,800 ton in 1986 to 561,000 ton in 2000 and average yields nationwide rose from $840-2,440 \mathrm{~kg} \mathrm{ha}^{-1}$ (DOF, 2005). With farm gate prices of farmed fish of about $\$ 0.80 \mathrm{~kg}^{-1}\left(\mathrm{Tk} 45-50 \mathrm{~kg}^{-1}\right)$, fresh water aquaculture production contributes about $\$ 700$ million year $^{-1}$ at farm gate value to the rural economy or $>\$ 1$ billion annually when postharvest handling and marketing are included. Although, identified as an emerging farming sector, aquaculture in Bangladesh is neglected due to several hindrances towards its growth and production and losing its revenue; infectious disease is one of major hindrances that have a negative impact on aquatic farming in Bangladesh.

The current trend in fisheries development is towards increased intensification and commercialization of their production. Like other farming sectors, the likelihood of major disease problems occurring increases as aquaculture activities intensify and expand. The fish farming industry has been overwhelmed with its share of diseases and problems caused by viruses, bacteria, fungi, parasites and other undiagnosed and emerging pathogens. Thus, infectious disease is now a primary constraint to the culture of many aquatic species, impeding both economic and social development.

The appearance and development of a fish or shrimp disease is the result of the interaction among pathogen, host and environment. Therefore, only multidisciplinary studies involving the characteristics of potential pathogenic microorganisms for fish and shrimp, aspects

Corresponding Author: Nilimesh Halder, Discipline of Computer Science and Engineering, University of Khulna, Khulna, Bangladesh 
of the transmission biology of the hosts as well as a better understanding of the environmental factors affecting such cultures will allow the application of adequate measures to prevent and control the main diseases limiting the production of freshwater fishes and shrimp in Bangladesh (BFRI, 2004).

It may be a general thought that statistical regression of either experimental or time series data is the only modeling technique available for the marine biologist's toolkit to analyze infectious disease epidemiology in large varieties of fish populations but statistical models are only one of several types of analytical models that are valuable to understand and predict transmission of infectious diseases. Mathematical modeling in the study of fish infectious diseases is a set of techniques, tools and equations that define interactions between individuals or populations and other individuals, populations and environments.

By defining the rules of these interactions and translating those rules into equations, a complex set of processes can be broken down into components and quantified. The model can then be used to explore relationships in the modeled populations, to examine the impact of changes of rules on the system and its components and the outcomes of various events that might have an effect on the population.

The foundations of mathematical modeling of human and mammalian disease epidemics were first established by Kermack and McKendrick $(1927,1932,1933)$. Later, the extensive reviews (Anderson, 1982; Anderson and May, 1979; May and Anderson, 1979) contributed to the subject of disease dynamics opening new discussions for concepts, critical threshold, basic reproductive ratio, transmission coefficient, etc. The importance of mathematical models in disease epidemics has been discussed extensively by many researchers (Bradley, 1982; Anderson and May, 1991; Heesterbeek and Roberts, 1995). A review (Ogut, 2001) on fish disease dynamics was also studied.

Considering the vast amount of experimental or time series data available about various aspects of fish pathogens, potentials of disease dynamics and epidemiological models should be explored. Since these types of studies focus on diseases rather than pathogen, the information is invaluable in terms of understanding and controlling disease. In the study, we focus on formulating a basic SIR model for fish and shrimp infectious disease in Bangladesh. We derive analytical model of fish infectious diseases and then analyze the outcomes of the model with numerical examples. In the following sections, we shall describe a compartmental modeling approach that captures the transmission dynamics of infectious diseases in aquatic populations as well as give a detail description of mass action theory in infectious disease transmission, basic reproduction number, transmission co-efficient and critical population densities for infectious disease epidemiology. Then, we shall give a theoretical framework that describes the stability and existence of an epidemic in a closed aquatic population. Finally, some numerical simulations from this model will be summarized with concluding remarks.

\section{MATERIALS AND METHODS}

Compartmental modeling of infectious disease dynamics:

A compartmental model of infectious disease dynamics is a model in which members of a host population are assigned to compartments on the basis of their infectious status or other attributes and the changes in the size of compartments are described as a dynamic system. The basic model of such kind is also known as SIR model with birth and death rate which is first stated by (Kermack and McKendrick, 1927).

The whole population $\mathrm{N}$ of an SIR model is subdivided in three different sub-classes as the Susceptible class S, the Infected class I and the Recovered or removed class $R$. The Susceptible class $S$ are those individuals who are not infective but who are capable of contracting the disease and becoming infective. The infective class I are those individuals who are infected with pathogen and capable of transmitting the disease to others. The Recovered or removed class $\mathrm{R}$ are those individuals who have had the disease and died or had the disease and recovered and/or become permanently immune and/or been isolated from the susceptible until recovery and permanent immunity occur. The dynamics of infectious disease transmission between the classes are shown in Fig. 1.

In the disease transmission model, we assume that aquatic population will be in closed form i.e., there is no birth and death. Initially whole population would be susceptible with a very few infected as introduced population. We also assume that all infected population will be recovered after certain period of time. Therefore, the simplified model that we use is shown in Fig. 2.

It is assumed that in directly transmitted diseases, the dynamics proceed according to the mass action theory, namely that the net rate of hosts becoming infected is the product of density of $\mathrm{S}$ individuals times the density of I individuals. Thus, the number of successful contacts made between $\mathrm{S}$ and I (infectious) determines the magnitude of the disease outcome. To construct the disease transmission model, we further assume that: 


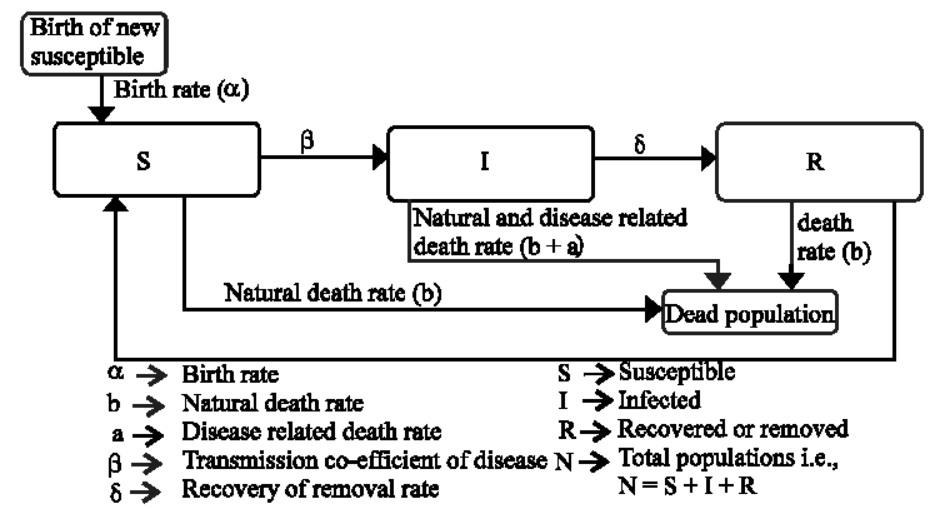

Fig. 1: Dynamics of infectious disease transmission between the SIR classes

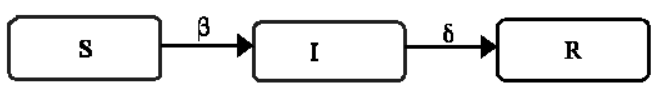

Fig. 2: Simplified SIR model for infectious disease

1: An average member of the population makes contact sufficient to transmit infection with $\beta \mathrm{N}$ others per unit time where $\mathrm{N}$ represents total population size (mass action incidence)

2: Infectives leave the infective class at rate $\delta \mathrm{I}$ per unit time

3: There is no entry into or departure from the population except possibly through death from the disease

According to point 1 , since the probability that a random contact by an infective is with a susceptible who can then transmit infection is $\mathrm{S} / \mathrm{N}$, the number of new infections in unit time per infective is $(\beta N)(\mathrm{S} / \mathrm{N})$, giving a rate of new infections $(\beta \mathrm{N})(\mathrm{S} / \mathrm{N}) \mathrm{I}=\beta \mathrm{S} I$. Alternately, the probability that this contact is with an infective is $\mathrm{I} / \mathrm{N}$ and thus the rate of new infections per susceptible is $(\beta \mathrm{N})$ $(\mathrm{I} / \mathrm{N})$ giving a rate of new infections $(\beta \mathrm{N})(\mathrm{I} / \mathrm{N}) \mathrm{S}=\beta \mathrm{SI}$. Therefore, the systems of differential equations that can describe the dynamics of infectious disease transmission in a fully susceptible closed aquatic population are given as follows:

$$
\begin{gathered}
\mathrm{dS} / \mathrm{dt}=-\beta \mathrm{SI} \\
\mathrm{dI} / \mathrm{dt}=\beta \mathrm{SI}-\delta \mathrm{I} \\
\mathrm{dR} / \mathrm{dt}=\delta \mathrm{I} \\
\mathrm{N}=\mathrm{S}+\mathrm{I}+\mathrm{R}
\end{gathered}
$$

Infectious disease transmission co-efficient $\beta$ is determined by two factors (Anderson, 1982), the frequency of contacts made which is directly related to the density of susceptible individuals and the likelihood that a contact results in pathogen transmission. In aquatic environment, all of the fish are exposed to the same environmental conditions. Since, it is a closed environment, making an assumption on contact rate that every fish has the same chance of coming into a contact with an infectious fish and/or an infectious agent in the water column is more than an assumption.

There is a threshold density which disease cannot persist. Kermack and McKendrick (1927) first reported the concept of threshold density. The critical threshold density in a closed population for a short period of time where no birth and death is considered is determined by the following relationship:

$$
\mathrm{N}_{\mathrm{T}}=\delta / \beta
$$

For a disease outbreak to occur, there should be a certain number of susceptible individuals in the population, higher than $\mathrm{N}_{\mathrm{T}}$ (i.e., $\mathrm{S}>\mathrm{N}_{\mathrm{T}}$ ) (Anderson and May, 1986; Nasel, 1995; Dietz, 1974).

For directly transmitted macro-parasites such as viral and bacterial pathogens, $\mathrm{N}_{\mathrm{T}}$ is low due to the production of large numbers of macro-parasites from individual hosts and the fact that parasites often have long infective stages which clearly indicates dependence of net rate of infected hosts on a certain critical density.

The number of I (infected) will not increase unless $\mathrm{N}>\mathrm{N}_{\mathrm{T}}$ (Anderson and May, 1986). With the exception of sexually transmitted diseases (Yorke et al., 1978), the requirement of a certain number of individuals for a disease epidemic is true for virtually all diseases (Anderson and May, 1979). The basic reproductive rate $\left(\mathrm{R}_{0}\right)$ is the most crucial component of the disease dynamics modeling process. $\mathrm{R}_{0}$ is the mean number of individuals directly infected by an infectious case during 
the individuals' entire infectious period after entering a totally susceptible population (Anderson, 1982; Anderson and May, 1979; May and Anderson, 1979; Anderson and May, 1991).

$\mathrm{R}_{0}$ is determined by factors which are specific to the disease agent (its virulence), the host (the level of susceptibility which determines levels of latent period-the time required for an epidemic to occur) and environmental factors such as host density and behavior (Anderson, 1982). The measurement of $R_{0}$ is essential in the determination of the spread of the disease, since it is the measure of the disease invasion in the population (Heesterbeek and Roberts, 1995).

It determines whether an invasion will emerge after introducing the infected individual $\mathrm{s}$ into a totally susceptible population. $\mathrm{R}_{0}$ could take one of three possible values as:

$\mathrm{R}_{0}<1$ : $\quad$ Disease will disappear over time

$\mathrm{R}_{0}=1$ : Disease will be endemic and persistent with no large scale epidemic occurring

$\mathrm{R}_{0}=1$ : There will be an epidemic with elevated levels of disease related mortality

$\mathrm{R}_{0}$ can be estimated using the formula:

$$
\mathrm{R}_{0}=\frac{\beta}{\delta}
$$

Where:

$\beta=$ Disease transmission co-efficient

$\mathrm{S}(0)=$ Initial susceptible population

$\delta=$ Recovery or removal rate from infection

\section{RESULTS AND DISCUSSION}

Equilibrium and stability analysis of infectious disease epidemiology: Modeling infectious diseases demand that we investigate whether the disease spread could attain an epidemic level or it could be wiped out. The equilibrium analysis helps to achieve this. Thus, two equilibriums will be considered; the disease free equilibrium and epidemic equilibrium. At equilibrium, the LHS of the three equations constituting the system of equations are zeros i.e., Eq. 1-3 will be zero as follows:

$$
\frac{\mathrm{dS}}{\mathrm{dt}}=0, \frac{\mathrm{dI}}{\mathrm{dt}}=0, \frac{\mathrm{dR}}{\mathrm{dt}}=0
$$

Hence, the system of equation becomes:

$$
-\beta \mathrm{SI}=0
$$

$$
\begin{gathered}
\beta S \mathrm{I}-\delta \mathrm{I}=0 \\
\delta \mathrm{I}=0
\end{gathered}
$$

From Eq. 5, we get either $\mathrm{S}=0$ or $\mathrm{I}=0$. If $\mathrm{S}=0$ then epidemic equilibrium:

$$
\left(\mathrm{S}^{*}, \mathrm{I}^{*}, \mathrm{R}^{*}\right)=\left(0, \mathrm{I}^{*}, 0\right)
$$

If $\mathrm{I}=0$, then disease free equilibrium:

$$
\left(\mathrm{S}^{*}, \mathrm{I}^{*}, \mathrm{R}^{*}\right)=\left(\mathrm{S}_{0}, 0,0\right)
$$

Where $S_{0}$ is the initial susceptible populations. To test for the stability of each of the two equilibriums where we examine the behavior of the model population near the equilibrium solutions (the disease free and the endemic), we need to linearize system of Eq. 1-3 by taking the Jacobian matrix of the system. For the modeled system of equation, the Jacobian matrix is:

$$
\mathrm{J}=\left[\begin{array}{ccc}
-\mathrm{BI} & -\mathrm{BS} & 0 \\
\mathrm{BI} & \mathrm{BS}-\delta & 0 \\
0 & \delta & 0
\end{array}\right]
$$

At the disease free equilibrium, $\left(\mathrm{S}_{0}, 0,0\right)$, the Jacobian matrix becomes:

$$
\mathrm{J}^{0}=\left[\begin{array}{ccc}
-\mathrm{BI} & -\mathrm{BS}_{0} & 0 \\
\mathrm{BI} & \mathrm{BS}^{0}-\delta & 0 \\
0 & \delta & 0
\end{array}\right]
$$

From 9 we have:

$$
\operatorname{det}\left(\mathrm{J}^{0}-\lambda \mathrm{I}\right)=\lambda^{2}(\lambda-\beta \mathrm{S}+\delta)
$$

Hence, the eigenvalues are:

$$
\lambda_{1}=\lambda_{2}=\text { and } \lambda_{3}=\beta \mathrm{S}-\delta
$$

Since, $\lambda_{3}$ is negative if $\delta>\beta S$, then it is stable in disease free equilibrium.At the epidemic equilibrium $\left(0, I^{*}\right.$, 0 ) the Jacobian matrix becomes:

$$
\mathrm{J}\left[\begin{array}{lll}
-\mathrm{BI} & 0 & 0 \\
\mathrm{BI} & -\delta & 0 \\
0 & \delta & 0
\end{array}\right]
$$

From 9 we have:

$$
\operatorname{det}\left(\mathrm{J}^{*}-\lambda \mathrm{I}\right)=(\lambda+\beta \mathrm{I})(\lambda+\delta)
$$


Since all eigenvalues of the epidemic equilibrium are negative, it is asymptotically stable. It follows that in a short outbreak, epidemic would occur within the closed population.

Numerical simulations: The system of differential Eq. 1-3 for understanding aquatic infectious disease epidemiology can be solved numerically using Berkeley Madonna v8.13 package. The hypothetical value of each parameter of the model is shown in Table 1 . The results from numerical simulations are shown in Fig. 3-5.

In Fig. 3, the dynamics of infectious disease progression is shown. With a small proportion of infected, the epidemic is occurred in the whole susceptible population within a very short period of time. Simulation results show that initially the infected appears in an exponential growth. Then the epidemic dies out due to lack of susceptible population.

The critical density of susceptible population is very important to carry on a disease. The critical $\mathrm{N}_{\mathrm{T}}$ density is equivalent to $\delta / \beta$. If susceptible population is $<\delta / \beta$ then the disease will not persist. This phenomenon is shown in Fig. 4.

The number of infected population is largely depended on the transmission coefficient of disease. In Fig. 5 impact of different transmission coefficients are shown. Mathematical modeling of aquatic infectious diseases has several potential benefits. For example, two fundamental assumptions in any infectious disease

Table 1: Hypothetic values of each model parameters used for numerical simulations

\begin{tabular}{ll}
\hline Parameters & Values \\
\hline I & 0.001 \\
S & $1-100$ \\
$\beta$ & 0.40 \\
$\delta$ & 0.05 \\
\hline
\end{tabular}

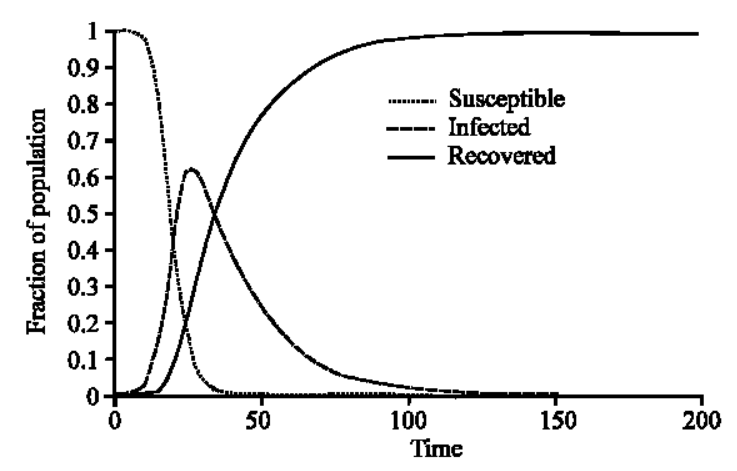

Fig. 3: Dynamics of disease progression in entirely susceptible populations modeling are homogeneous mixing and the closed populations that make model robust in terms of predictive capabilities. In pond and cage culture situations, environment is homogenous for each individual sharing the same environment and the population can also be considered as in closed form.

Disease epidemic is the result of the complex combination of environmental, host and pathogen related factors. Therefore, environmental (temperature, oxygen, pollutions), host (species, age, density, immunity) or pathogen related factors (virulence, strain) could be manipulated to control diseases.

It is a fact that the system is very complex, however not all parameters have the same amount of effect on the outcome of the disease. Some parameters playing a role in disease dynamics could be key factors. Adjusting just those key factors could make significant improvement in the system in terms of decreasing loses or prevalence. In Fig. 1, we showed the complex effect of disease transmission co-efficient on development of an epidemic in the susceptible populations. Modeling disease

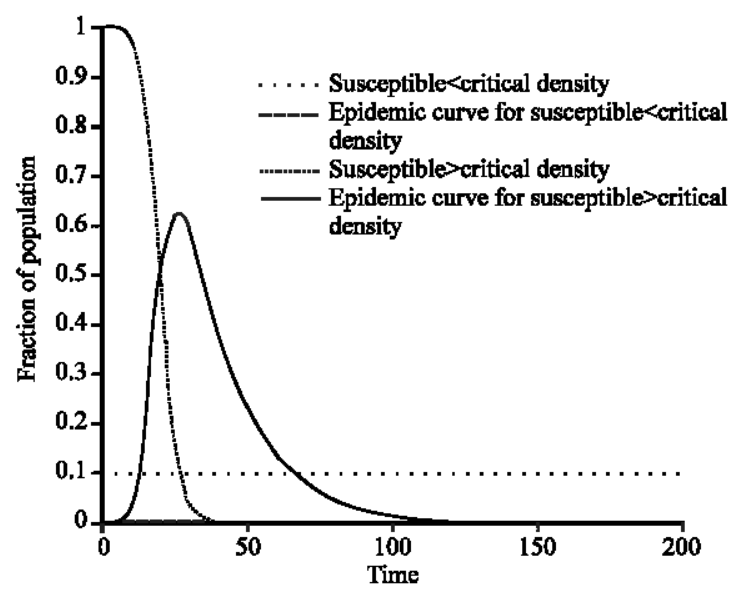

Fig. 4: Critical population density to persist an epidemic

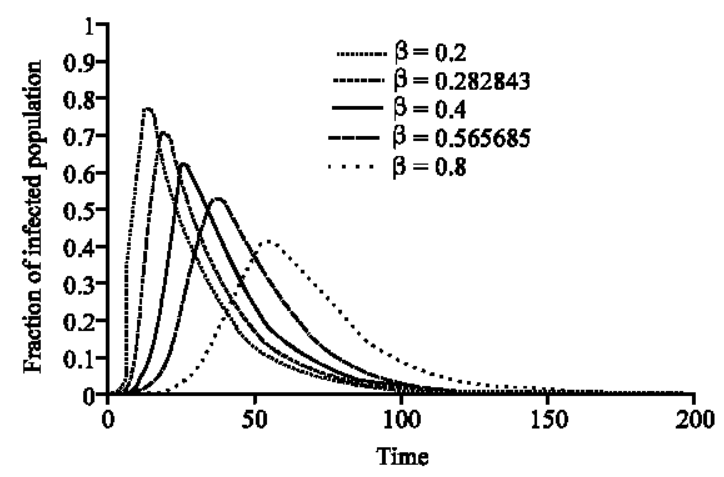

Fig. 5: Changes in fraction of infected population due to varying disease transmission coefficient values 
dynamics totally focuses on disease by estimating $R_{0}$ and $N_{T}$ values and observes changes on these parameters. Any significant decrease in these values indicates effectiveness of a control measure which could be a drug treatment or vaccination.

Here, controlling the disease means pulling $\mathrm{R}_{0}$ below unity. Therefore, there may not be any need to vaccinate the whole population but just enough proportion to draw $\mathrm{R}_{0}$ below unity. This will decrease cost and effort of controlling the disease.

\section{CONCLUSION}

In brief, the large amounts of data have been accumulated on various pathogens of fish in Bangladesh (BFRI, 2004; DOF, 2005). However, the list of things that we do not know includes many issues of fundamental, practical and theoretical importance about aquatic disease. The main reason for this gap is that the disease itself has not been in focus but pathogen. Mathematical modeling is one approach studying disease itself and guides us to take necessary steps in controlling and preventing diseases.

\section{REFERENCES}

Anderson, R.M. and R.M. May, 1979. Population biology of infectious diseases: Part I, Nature, 280: 361-367.

Anderson, R.M., 1982. The Population Dynamics of Infectious Diseases: Theory and Applications. Chapman and Hall, London.

Anderson, R.M. and R.M. May, 1986. The invasion, persistence and spread of infectious diseases within animal and plant communities. Philosophical Trans. R. Soc. London B, 314: 533-568.

Anderson, R. and R. May, 1991. Infectious Diseases of Humans: Dynamics and Control. Oxford University Press, Oxford.

BBS, 2000a. Household Expenditure Survey. BBS, Dhaka. BBS, 2000b. Statistical Yearbook of Bangladesh. BBS, Dhaka.

BFRI, 2004. Annual report 2004-2005. Bangladesh Fisheries Research Institute. http:/www.fri.gov.bd/.
Bradley, D.J., 1982. Epidemiological Models-Theory and Reality. In: Population Dynamics of Infectious Diseases, Anderson, R.M. (Ed.). Chapman and Hall, London, pp: 320-334.

DOF, 2005. Annual report 2005-2006. Department of Fisheries, Ministry of Fisheries and Livestock, Dhaka, Bangladesh.

Dietz, K., 1974. Transmission and control of arbovirus diseases. Proceedings of Society for Industrial and Applied Mathematics, July 8-12, Philadelphia, pp: 104-121.

Heesterbeek, J.A.P. and M.G. Roberts, 1995. Mathematical Models for Microparasites of Wildlife. In: Ecology of Infectious Diseases in Natural Populations, Grenfell, B.T. and A.P. Dobson (Eds.). Cambridge University Press, Cambridge, pp: 91-122.

Kermack, W.O. and A.G. McKendrick, 1927. Contribution to the mathematical theory of epidemics, part I. Proc. R. Soc. London Ser. A, 115: 700-721.

Kermack, W.O. and A.G. McKendrick, 1932. Contribution to the mathematical theory of epidemics, II- The problem of endemicitY. Proc. R. Soc. London Ser. A, 138: $55-83$.

Kermack, W.O. and A.G. McKendrick, 1933. Contribution to the mathematical theory of epidemics, III- Further studies of the problem of endemicity. Proc. R. Soc. London Ser. A, 141: 94-122.

May, R.M. and R.M. Anderson, 1979. Population biology of infectious diseases: Part II, Nature, 280: 455-461.

Nasel, I., 1995. The Threshold Concept in Deterministic and Stochastic Models. In: Epidemic Models: Their Structure and Relation to Data, Mollison, D. (Ed.). Newton Institute, USA., pp: 71-83.

Ogut, H., 2001. Modeling of fish disease dynamics: A new approach to an old problem. Turk. J. Fish. Aquat. Sci., 1: 67-74.

Thompson, P., N. Roos, P. Sultana and S.H. Thilsted, 2002. Changing significance of Inland fisheries for livelihoods and nutrition in Bangladesh. J. Crop Prod., 6: 249-317.

Yorke, J.A., H.W. Hethcote and A. Nold, 1978. Dynamic and control of the transmission of gonorrhea. Sexually Transmitted Dis., 5: 51-56. 